\title{
Epstein-Barr Virus (EBV) infection related thrombocytosis : A Case Report
}

\author{
Suman Adhikari, Rewati Raman Malla, Manoj shah, Ananta Upreti, Bhawesh Thapa
}

Department of Internal Medicine, National Academy of Medical Sciences (NAMS), Bir Hospital Kathmandu, Nepal

DOI: https://doi.org/10.3126/jaim.v7i2.23491

Keywords: Epstein-Barr Virus(EBV), infection, thrombocytosis,

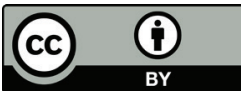

This work is licensed under a Creative Commons Attribution 3.0 Unported License.

\begin{abstract}
EBV infection can occur worldwide.We managed a 17 year old male who developed transient pancytopenia followed by thrombocytosis concurrent with EBV infection. To the best of our knowledge we have not encountered any cases of thrombocytosis concurrent with EBV infection before.Our case was diagnosed as a case of EBV infection with the help of serology and managed conservatively. In our case,though it is uncommon to find thrombocytosis due to EBV infection,it is possible that following pancytopenia concurrent with the EBV infection, the patient might have developed secondary or reactive thrombocytosis due to elevated endogenous levels of thrombopoietin, interleukin-6, catecholamines.
\end{abstract}

\section{Introduction}

EBV infection can occur in any part of the world and infection is common in early childhood and the second peak can occur during late adolescence, most of the EBV infections in infants and young children are asymptomatic. Whereas around $75 \%$ of infections in adolescents may present with infectious mononucleosis, commom symptoms of this infection are sore throat, malaise, headache, abdominal pain and common signs are lympadenopathy, fever, pharyngitis, hepatomegaly, splenomegaly, rash. ${ }^{1}$ Some adults (less than $0.5 \%$ ) have been reported to have life-threatening hematological complications like subcapsular splenic hemorrhage or splenic rupture. Other hematological complications : Coombs test positive hemolytic anemia, severe thrombocytopenia, neutropenia, and aplastic anemia. ${ }^{2}$ Many cases of ITP turning to aplastic anemia have been found in the literature. ${ }^{3,4} \operatorname{IgM}$ and $\operatorname{IgG}$ antibodies directed against the Epstein-Barr viral capsid antigen (VCA) are usually present at the onset of clinical illness because of the long viral incubation period. IgM levels wane approximately three months later; thus, they are a good marker of acute infection. IgG VCA antibodies persist for life and are a marker of EBV infection. ${ }^{5}$

\section{Case Report:}

A seventeen year-old male presented with complaints of easy fatigability of 4 months duration,abdominal pain of 1 months duration and fever for 5 days. He had decreased energy associated with fatigue, decreased appetite. His sister also noticed pallor. The easy fatigability was progressive and was associated with palpitation on mild exertions because of which he had to take rest. His abdominal pain was mainly over epigastrium and left upper quadrant of abdomen and was dull aching in type associated with nauseating feeling and loss of apetite and occasional vomitings.
The fever was intermittent not associated with chills and sweating,with maximum documented temperature being 102 degree F.He presented to our centre with those complaints.

He had no significant past medical history. He was not taking any medications except some iron tablets for anemia from a nearby health facility and had no known allergies.

Physical examination showed a febrile pale and ill-looking young male with normal other vital signs. Per-abdominal findings were suggestive of palpable nontender spleen around $2 \mathrm{~cm}$ below left subcostal margin along mid clavicular line,otherwise no other significant systemic findings were noted.

Initial laboratory results showed WBC: 2800 per cumm (neutrophils $20 \%$, lymphocytes $79 \%$, monocytes $0 \%$, eosinophils 1\%), Hb: 6.4 g/dL, Hct: $17 \%$, platelets: $59000 / \mu L$, RBC: 1.91 million/ $\mu \mathrm{L}, \mathrm{MCV}$ : 91 , Serum ferritin 382(13-400 ng/ml), Serum iron $124 \mathrm{mcg} / \mathrm{dl}(65-175 \mu \mathrm{g} / \mathrm{dL})$, TIBC $279 \mathrm{mcg} / \mathrm{dl}$ (228 -428mcg/ $\mathrm{dl})$, iron saturation $44 \%$ (21-42\%). Serum folate $3.9 \mathrm{ng} / \mathrm{ml}(5.2-$ $20)$ and $\mathrm{B} 12-765.4 \mathrm{pg} / \mathrm{ml}(200-1100 \mathrm{pg} / \mathrm{ml})$ and Reticulocyte of $0.8 \%$.

Peripheral smear showed macrocytic,microcytic and hypochromic occasional fragmented RBCs, decreased WBC with relative lymhocytosis, no blast, and reduced platelets without atypical cells and Chest $\mathrm{X}$-ray was normal.

\section{Correspondence Author}

Dr. Suman Adhikari,

Department of Internal Medicine

NAMS, Bir Hospital, Kathmandu, Nepal

Phone no.-9846022044,

Email-adhikari11613@gmail.com 
The fragmented RBCs in smear could be due to hemolytic anemia caused by EBV infection itself. The bone marrow biopsy and aspiration showed hypercellular marrow with erythroid hyperplasia with mixed erythroid maturation with increased megakaryopoiesis, this could be possible because the patient presented to us probably during recovery phase from acute infection.USG abdomen showed borderline splenomegaly, and antiHCV,HBsAG and HIV,anti-HAV,HEV were nonreactive,Direct Coombs Test- negative,scrub typhus, brucella and leptospira-antibodies negative, mantoux test negative, serum total bilirubin- $1.5 \mathrm{mg} / \mathrm{dl}$ with direct component $-0.7 \mathrm{mg} / \mathrm{dl}$,SGPT36U/1,SGOT-38U/1,ALP-43U/1,serum LDH-1536U/1 ,total protein- $6.4 \mathrm{~g} / \mathrm{dl}$,albumin- $4.5 \mathrm{~g} / \mathrm{dl}$, calcium- $8.6 \mathrm{mg} / \mathrm{dl}$,phosphorus$4.3 \mathrm{mg} / \mathrm{dl}$,stool for occult blood-negative,K39-negative,RA factor-negative,ANA-negative,malarial antigen negative and haptoglobin- $0.5 \mathrm{mg} / \mathrm{dl}$ (decreased). The high LDH,decreased haptoglobin could be parts of hemolysis concurrent with EBV infection.

The EBV(VCA) IgG=4.76(positive if $>1.1$ index value) and $\operatorname{IgM}=1$ (equivocal if $0.9-1.1$,positive if more than 1.1 , negative if less than 0.9 index value).

The patient was given blood transfusion for anemia following supportive treatment,after about 7 days of admission he developed thrombocytosis,with maximum platelates count reaching upto $10,5900 / \mathrm{mm} 3$ which fell on its own to normal range within a period of 1 week,during which he was carefully observed for possible complications.He didn't develop any complications of thrombocytosis.After 2 weeks of in-hospital medical management his Hb-13.8 g/dl,total count-7700/mm3 with neutrophils-67\%lymphocytes- $31 \%$ and eosinophils$2 \%$,platelets-527,000/mm3.He was discharged and asked for follow up in 2 weeks. Thus our patient was diagnosed with the help of EBV VCA and managed with supportive treatment and he recovered.

\section{Discussion:}

Aplastic anemia after primary EBV infection or reactivation of EBV infection is enumerated very well in the literature, but thrombocytosis following EBV infection has not been documeneted. Our case is development of transient pancytopenia which later on was followed by thrombocytosis apparently following EBV infection.We think this case could be one of the rarest cases of $\mathrm{EBV}$ infection causing thrombocytosis.

The most common cause of thrmbocytosis is a reactive process. The platelet count as such can not differentiate primary from reactive thrombocytosis.In one study with series of 732 patients with platelet counts 500,000 per cubic millimeter or higher,around $88 \%$ had secondary thrombocytosis, the most common causes were major surgery,infection, cancer and chronic inflammation. ${ }^{6}$

Likewise,in another series of 280 hospitalized patients with platelet count of 1 million per cubic millimeter or higher,around $82 \%$ cases had reactive thrombocytosis. ${ }^{7}$

Reactive thrombocytosis can be caused by increased endogenous levels of thrombopoietin,IL-6 and catecholamines. These mediators are produced because of inflammation,infection, and neoplastic conditions or situations causing stress. The levels of thrombopoietin are high in reactive thrombocytosis. ${ }^{8,9}$ Elevated plasma levels of IL-6 plays an important role in acute phase response of inflammatory and neoplastic processes.IL-6 increases the expression of thrombopoeitin messanger RNA in the liver. ${ }^{10}$

Thus, IL-6 may be a key mediator of the increased synthesis of thrombopoietin and the consequent reactive thrombocytosis. ${ }^{11}$

\section{Conclusion}

In our case,it is possible that following pancytopenia concurrent with the EBV infection,the patient might have developed thrombocytosis but it's difficult to say whether it's clonal(primary) or reactive type but the scenario may favour reactive type which in turn may be speculated to be caused by elevated endogenous levels of thrombopoietin, interleukin-6, other cytokines, or catecholamines which are not measured in this case.This could well be a tug of war between the myelosuppressive effects of EBV itself and the increasing levels of endoghenous mediators during recovery phase of the infection.

\section{References:}

1 Jeffrey 1. Cohen, 2016, Epstein-Barr Virus infections, including infectious mononucleosis, Harrisons Principle of Internal Medicine 19th Edition, Section 8, Chapter 218.

2. Jenson HB, Epstein-Barr Virus. In Kliegman RM, Nelson Textbook of Pediatrics. 20th edition. ElsevierSaunders.2016,1586-1590.

3. Weinblatt ME. Immune thrombocytopenic purpura evolving into aplastic anemia in association with Epstein-Barr virus infection. Journal of Pediatric Hematology/Oncology. 1991 Jan 1;13(4):465-9.

4. Baranski B, Armstrong G, Truman JT, et al. Epstein-Barr virus in the bone marrow of patients with aplastic anemia. Annals of internal medicine. 1988 Nov 1;109(9):695-704.

5. Obel N, HØIER-MADSEN MI, Kangro H. Serological and clinical findings in patients with serological evidence of reactivated Epstein-Barr virus infection. Apmis. 1996 Jan $1 ; 104(1-6): 424-8$.

6.Griesshammer M, Bangerter M, Sauer T, et al. Aetiology and clinical significance of thrombocytosis: analysis of 732 patients with an elevated platelet count. J Intern Med 1999; 245:295-300.

7. Buss DH, Cashell AW, O'Connor ML, et al. Occurrence, etiology and clinical significance of extreme thrombocytosis: a study of 280 cases. Am J Med 1994;96:247-53.

8. Wang JC, Chen C, Novetsky AD, et al. Blood thrombopoietin levels in clonal thrombocytosis and reactive thrombocytosis. Am J Med 1998; 104:451-5.

9. Hsu HC, Tsai WH, Jiang ML, et al. Circulating levels of thrombopoietic and inflammatory cytokines in patients with clonal and reactive thrombocytosis. J Lab Clin Med 1999;134:392-7.

10. Kaser A, Brandacher G, Steurer W, et al. Interleukin-6 stimulates thrombopoiesis through thrombopoietin: role in inflammatory thrombocytosis. Blood 2001;98:2720-5.

11.Schafer AI. Thrombocytosis and thrombocythemia. Blood Rev 2001;15:159-66 